\title{
On the Convergence of the Modified Riccati Equation
}

\author{
Nicholas Assimakis ${ }^{1}$ and Maria Adam² \\ ${ }^{1}$ Department of Electronics, Technological Educational Institute of Lamia, 35100 Lamia, Greece \\ ${ }^{2}$ Department of Computer Science and Biomedical Informatics, University of Central Greece, 35100 Lamia, Greece
}

Correspondence should be addressed to Maria Adam, madam@ucg.gr

Received 5 March 2012; Accepted 26 April 2012

Academic Editors: C.-W. Kok and C.-M. Kuo

Copyright (C 2012 N. Assimakis and M. Adam. This is an open access article distributed under the Creative Commons Attribution License, which permits unrestricted use, distribution, and reproduction in any medium, provided the original work is properly cited.

The modified Riccati equation arises in the implementation of Kalman filter in target tracking under measurement uncertainty and it cannot be transformed into an equation of the form of the Riccati equation. An iterative solution algorithm of the modified Riccati equation is proposed. A method is established to decide when the proposed algorithm is faster than the classical one. Both algorithms have the same behavior: if the system is stable, then there exists a steady-state solution, while if the system is unstable, then there exists a critical value of the measurement detection probability, below which both iterative algorithms diverge. It is established that this critical value increases in a logarithmic way as the system becomes more unstable.

\section{Introduction}

The discrete time modified Riccati equation emanating from Kalman filter was originally formulated in [1]. It plays an important role in target tracking [1-10]. Theoretical properties of the modified Riccati equation have been derived in $[2,3]$. It is well known [2] that the modified Riccati equation cannot be transformed into an equation of the form of the Riccati equation. The discrete time Riccati equation arises in linear estimation, namely, in the implementation of the discrete time Kalman filter [11]. The modified Riccati equation is solvable under certain conditions $[2,9]$ and has existence and uniqueness properties similar to the Riccati equation [2].

In Section 2, the modified Riccati equation associated with target tracking under measurement uncertainty is presented; the case without clutter but with detection probability of less than one is considered. In Section 3, an iterative solution algorithm of the modified Riccati equation is proposed and compared to the classical one. A method is established to distinguish the faster algorithm. If the system is stable, then both algorithms do converge to the steady-state solution. If the system is unstable, then there exists a critical value of the measurement detection probability, below which both algorithms diverge. In Section 4, it is established that this critical value increases in a logarithmic way as the system becomes more unstable.

\section{The Modified Riccati Equation}

Consider the following state space equations at time $k \geq 0$ :

$$
\begin{gathered}
x_{k+1}=F x_{k}+w_{k}, \\
z_{k}=H x_{k}+v_{k},
\end{gathered}
$$

where $x_{k}$ is the $n \times 1$ state vector at time $k, z_{k}$ is the $m \times 1$ measurement vector, $F$ is the $n \times n$ system transition matrix, and $H$ is the $m \times n$ output matrix. It is assumed that $\left\{w_{k}\right\}$ and $\left\{v_{k}\right\}$ are zero mean, independent, white, Gaussian noise processes with constant covariance matrices given by $E\left[w_{k} w_{k}^{T}\right]=Q$ and $E\left[v_{k} v_{k}^{T}\right]=R$, that is, $Q$ is the $n \times n$ plant noise covariance matrix $R$ is the $m \times m$ measurement noise covariance matrix.

At the initial time $k=0$, the state $x_{0}$ is independent of the processes $\left\{w_{k}\right\}$ and $\left\{v_{k}\right\}$ for any $k$ and $x_{0}$ is a Gaussian random variable with mean $\bar{x}_{0}$ and covariance $P_{0}$, that is,

$$
E\left[x_{0}\right]=\bar{x}_{0}, \quad E\left[\left(x_{0}-\bar{x}_{0}\right)\left(x_{0}-\bar{x}_{0}\right)^{T}\right]=P_{0} .
$$

For $k \geq 0$, denoting $Z_{k}=\left\{z_{0}, z_{1}, \ldots, z_{k}\right\}$, the state prediction $x_{k / k-1}=E\left[x_{k} / Z_{k-1}\right]$ and the prediction error covariance matrix $P_{k / k-1}=E\left[\left(x_{k}-x_{k / k-1}\right)\left(x_{k}-x_{k / k-1}\right)^{T} / Z_{k-1}\right]$, and using the discrete-time invariant Kalman filter equations as described in [11-13], we derive the following recursion 
TABLE 1: Calculation burden of matrix operations.

\begin{tabular}{lc}
\hline Matrix operation & Calculation burden \\
\hline$A(n \times n)+B(n \times n)=S$ symmetric & $(1 / 2)\left(n^{2}+n\right)$ \\
$c \cdot S(n \times n)$ symmetric & $(1 / 2)\left(n^{2}+n\right)$ \\
$A(n \times m) \cdot B(m \times k)=C(n \times k)$ & $2 n m k-n k$ \\
$A(n \times m) \cdot B(m \times n)=S$ symmetric & $n^{2} m+n m-(1 / 2)\left(n^{2}+n\right)$ \\
{$[A(n \times n)]^{-1}=B(n \times n)$} & $(1 / 6)\left(16 n^{3}-3 n^{2}-n\right)$ \\
\hline
\end{tabular}

for the symmetric $n \times n$ prediction error covariance matrix $P_{k+1 / k}$, the Riccati equation:

$$
\begin{aligned}
P_{k+1 / k}=Q & +F P_{k / k-1} F^{T} \\
& -F P_{k / k-1} H^{T}\left[H P_{k / k-1} H^{T}+R\right]^{-1} H P_{k / k-1} F^{T}
\end{aligned}
$$

with initial condition $P_{0 /-1}=P_{0}$.

The state space equations in (1) can be used in target tracking to describe a linear target motion and measurement model. In addition, consider that a measurement is received with detection probability $p_{d}[2]$, where

$$
0 \leq p_{d} \leq 1 .
$$

Using the Kalman filter equations we are able to derive $[2,3]$ a Kalman-like recursion for the symmetric $n \times n$ prediction error covariance matrix $P_{k+1 / k}$, the modified Riccati equation:

$$
\begin{aligned}
P_{k+1 / k}= & Q+F P_{k / k-1} F^{T} \\
& -p_{d} F P_{k / k-1} H^{T}\left[H P_{k / k-1} H^{T}+R\right]^{-1} H P_{k / k-1} F^{T} .
\end{aligned}
$$

Note that the prediction error covariance matrix is nonnegative definite $\left(P_{k+1 / k} \geq 0\right)$.

Also in the modified Riccati equation (5) note that the $m \times m$ matrix $H P_{k / k-1} H^{T}+R$ is nonsingular, when $R$ is a positive definite matrix $(R>0)$, which has the significance that no measurement is exact; this is reasonable in physical problems.

It is remarkable that the following special cases are implied by (5).

(i) Setting $p_{d}=1$, the classical Riccati equation (3) is derived. The difference between the modified Riccati equation and the Riccati equation is the term of detection probability $p_{d}$. It is obvious that the modified Riccati equation (5) cannot be transformed into the classical Riccati equation (3).

(ii) Setting $p_{d}=0$ in (5), the classical Lyapunov equation is derived:

$$
P_{k+1 / k}=Q+F P_{k / k-1} F^{T}
$$

which arises from the Riccati equation (3) in the infinite measurement noise case $(R \rightarrow \infty)$.

\section{Iterative Solutions of the Modified Riccati Equation}

Concerning the modified Riccati equation, it is known [9] that for stable systems, which means that all eigenvalues of $F$ lie inside the unit circle, the modified Riccati equation always converges and the limiting value $P$ of the prediction error covariance is the steady state solution of the discrete time modified Riccati equation.

The classical implementation of the modified Riccati Equation (cmRE) arises from (5), which consists of the direct implementation of the recursion of the following equation:

$$
\begin{aligned}
P_{k+1 / k}=F\left\{P_{k / k-1}-p_{d} F P_{k / k-1} H^{T}\right. & \\
& \left.\times\left[H P_{k / k-1} H^{T}+R\right]^{-1} H P_{k / k-1}\right\} F^{T}+Q .
\end{aligned}
$$

It is obvious that this equation is equivalent to the modified Riccati equation (5) achieving a reduction in computational burden by using as a common factor $F$.

Notice that if $R>0$, then the nonsingularity of $H P_{k / k-1} H^{T}+R$ in (7) is guaranteed.

It is known [2] that the steady state solution of the modified Riccati equation is independent of the initial condition $P_{0 /-1}=P_{0}$. So, for convenience, we are able to use zero initial condition $P_{0}=0$. Then we are able to use $P_{1 / 0}=Q$ as initial condition for the classical implementation.

Note that the convergence is achieved, when $\| P_{k+1 / k}-$ $P_{k / k-1} \|<\varepsilon$, where $\varepsilon$ is a small positive number and $\|M\|$ denotes the norm of the matrix $M$, which is equal to the largest singular value of $M$. Then the steady state solution $P$ satisfies the steady state modified Riccati equation:

$$
P=Q+F P F^{T}-p_{d} F P H^{T}\left[H P H^{T}+R\right]^{-1} H P F^{T} .
$$

The proposed implementation of modified Riccati Equation (pmRE) consists of the direct implementation of the recursion of the following equation:

$$
\begin{aligned}
P_{k+1 / k}= & \left(1-p_{d}\right)\left(Q+F P_{k / k-1} F^{T}\right) \\
& +p_{d}\left(Q+F\left[P_{k / k-1}^{-1}+H^{T} R^{-1} H\right]^{-1}\right) F^{T} .
\end{aligned}
$$

This equation is equivalent to the modified Riccati equation (5) and can be derived from (5) using the matrix inversion lemma, under the condition that for $k \geq 1$ the prediction error covariance matrix $P_{k / k-1}$ is positive definite $\left(P_{k / k-1}>\right.$ $0)$. This is guaranteed, if $Q>0$, due to the fact that $P_{1 / 0}=Q$, if we use zero initial condition $P_{0 /-1}=P_{0}=0$.

Notice that if $R>0$, then the existence of $H^{T} R^{-1} H$ in (9) is guaranteed and the nonsingularity of $P_{k / k-1}^{-1}+H^{T} R^{-1} H$ becomes obvious. Also note that $H^{T} R^{-1} H$ in (9) is computed once (initialization process).

Both the classical and the proposed algorithms for solving the modified Riccati equation are recursive ones. Thus, the total computational time required for the implementation of each algorithm is

$$
t_{a}=B_{a} S_{a} t_{o}
$$


TABLE 2: Classical implementation of the modified Riccati equation (cmRE).

\begin{tabular}{lcc}
\hline Matrix operation & Matrix dimensions & Calculation burden \\
\hline$H \cdot P_{k / k-1}$ & $(m \times n) \cdot(n \times n)$ & $2 n^{2} m-n m$ \\
$H P_{k / k-1} \cdot H^{T}$ & $(m \times n) \cdot(n \times m)^{*}$ & $n m^{2}+n m-(1 / 2)\left(m^{2}+m\right)$ \\
$O_{k} \equiv H P_{k / k-1} H^{T}+R$ & $(m \times m)+(m \times m)^{*}$ & $(1 / 2)\left(m^{2}+m\right)$ \\
$O_{k}^{-1}$ & $(m \times m)$ & $(1 / 6)\left(16 m^{3}-3 m^{2}-m\right)$ \\
$K_{k} \equiv O_{k}^{-1} \cdot H P_{k / k-1}$ & $2 n m^{2}-n m$ \\
$W \equiv P_{k / k-1} H^{T} \cdot K_{k}$ & $(m \times m) \cdot(m \times n)$ & $n^{2} m+n m-(1 / 2)\left(n^{2}+n\right)$ \\
$p_{d} \cdot W$ & $(n \times m) \cdot(m \times n)^{*}$ & $(1 / 2)\left(n^{2}+n\right)$ \\
$\Pi_{1} \equiv P_{k / k-1}-p_{d} \cdot W$ & $(n \times n)^{*}$ & $(1 / 2)\left(n^{2}+n\right)$ \\
$F \cdot \Pi_{1}$ & $(n \times n)+(n \times n)^{*}$ & $2 n^{3}-n^{2}$ \\
$\Pi_{2} \equiv F \Pi_{1} \cdot F^{T}$ & $(n \times n) \cdot(n \times n)$ & $n^{3}+(1 / 2)\left(n^{2}-n\right)$ \\
$P_{k+1 / k}=Q+\Pi_{2}$ & $(n \times n) \cdot(n \times n)^{*}$ & $(1 / 2)\left(n^{2}+n\right)$ \\
\hline
\end{tabular}

*Symmetric matrix.

TABLE 3: Proposed implementation of the modified Riccati equation (pmRE).

\begin{tabular}{|c|c|c|}
\hline Matrix operation & Matrix dimensions & Calculation burden \\
\hline$P_{k / k-1}^{-1}$ & $(n \times n)$ & $(1 / 6)\left(16 n^{3}-3 n^{2}-n\right)$ \\
\hline$M \equiv P_{k / k-1}^{-1}+H^{T} R^{-1} H$ & $(n \times n)+(n \times n)^{*}$ & $(1 / 2)\left(n^{2}+n\right)$ \\
\hline$M^{-1}$ & $(n \times n)$ & $(1 / 6)\left(16 n^{3}-3 n^{2}-n\right)$ \\
\hline$F \cdot M^{-1}$ & $(n \times n) \cdot(n \times n)$ & $2 n^{3}-n^{2}$ \\
\hline$F M^{-1} \cdot F^{T}$ & $(n \times n) \cdot(n \times n)^{*}$ & $n^{3}+(1 / 2)\left(n^{2}-n\right)$ \\
\hline$\Omega \equiv Q+F M^{-1} F^{T}$ & $(n \times n)+(n \times n)^{*}$ & $(1 / 2)\left(n^{2}+n\right)$ \\
\hline$p_{d} \cdot \Omega$ & $(n \times n)^{*}$ & $(1 / 2)\left(n^{2}+n\right)$ \\
\hline$F \cdot P_{k / k-1}$ & $(n \times n) \cdot(n \times n)$ & $2 n^{3}-n^{2}$ \\
\hline$F P_{k / k-1} \cdot F^{T}$ & $(n \times n) \cdot(n \times n)^{*}$ & $n^{3}+(1 / 2)\left(n^{2}-n\right)$ \\
\hline$\Psi \equiv Q+F P_{k / k-1} F^{T}$ & $(n \times n)+(n \times n)^{*}$ & $(1 / 2)\left(n^{2}+n\right)$ \\
\hline$\left(1-p_{d}\right) \cdot \Psi$ & $(n \times n)^{*}$ & $(1 / 2)\left(n^{2}+n\right)$ \\
\hline$P_{k+1 / k}=\left(1-p_{d}\right) \Psi+p_{d} \Omega$ & $(n \times n)+(n \times n)^{*}$ & $(1 / 2)\left(n^{2}+n\right)$ \\
\hline \multicolumn{3}{|c|}{$B_{\mathrm{pmRE}}=(1 / 3)\left(34 n^{3}+3 n^{2}+5 n\right)$} \\
\hline
\end{tabular}

${ }^{*}$ Symmetric matrix.

where $B_{a}$ is the per recursion calculation burden required for the online calculations of each algorithm, $S_{a}$ is the number of recursions (steps) that each algorithm executes, and $t_{o}$ is the time required to perform a scalar operation.

Note that the two algorithms are equivalent to each other with respect to their behaviour: they calculate theoretically such steady-state prediction error variance $P$ that satisfies (8). Then, it is reasonable to assume that both algorithms compute the limiting solution of the modified Riccati equation executing the same number of recursions, depending on the desired accuracy. Thus, in order to compare the algorithms with respect to their computational time, we have to compare their per recursion calculation burden required for the online calculations; the calculation burden of the offline calculations (initialization process) is not taken into account.

The computational analysis is based on the calculation burden of the matrix operations, which are summarized in Table 1 and needed for the implementation of the filtering algorithm [14].
Then, the (per recursion) computational requirements of the classical and the proposed algorithms for solving the modified Riccati equation are computed as

$$
\begin{aligned}
B_{\mathrm{cmRE}}= & \frac{1}{2}\left(6 n^{3}+n^{2}+n\right) \\
& +3 n^{2} m+3 n m^{2}+\frac{1}{6}\left(16 m^{3}-3 m^{2}-m\right), \\
B_{\mathrm{PmRE}}= & \frac{1}{3}\left(34 n^{3}+3 n^{2}+5 n\right) .
\end{aligned}
$$

The details of (11) are given in Tables 2 and 3.

From the above computational requirements where we derive the following conclusions.

(1) The per recursion calculation burden of the classical algorithm depends on the state dimension $n$ and on the measurement dimension $m$, while the per recursion calculation burden of the proposed algorithm depends only on the state dimension $n$. 


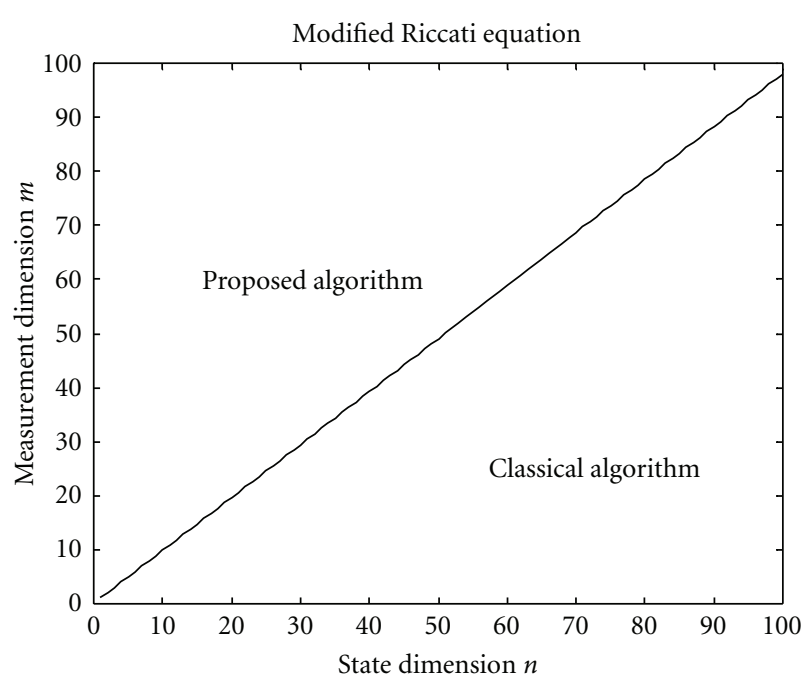

Figure 1: Proposed algorithm may be faster than the classical one.

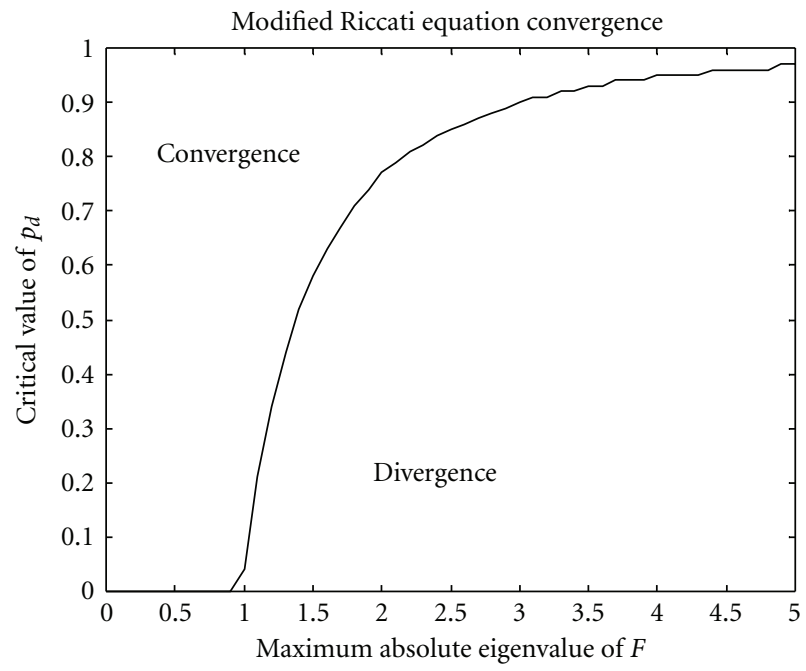

FIGURE 2: Modified Riccati equation convergence with respect to system stability.

(2) The proposed algorithm is faster than the classical one if the following relation holds:

$$
\begin{aligned}
B_{\mathrm{cmRE}}-B_{\mathrm{pmRE}}= & 3 n^{2} m+3 n m^{2}+\frac{1}{6}\left(16 m^{3}-3 m^{2}-m\right) \\
& -\frac{1}{6}\left(50 n^{3}+3 n^{2}+7 n\right)>0 .
\end{aligned}
$$

Figure 1 depicts the relation between the dimensions $n$ and $m$ that may hold in order to decide which algorithm is faster. Then, relation (12) is approached by the relation:

$$
m>n \text {. }
$$

Thus it becomes obvious that we are able to establish the following method to distinguish the faster algorithm:

"if $m>n$, then the proposed algorithm is faster than the classical algorithm, else the classical algorithm is faster than the proposed algorithm".

\section{Convergence of the Modified Riccati Equation}

Concerning the modified Riccati equation, it is known [9] that, for unstable systems (there is at least one eigenvalue of $F$ that lies strictly outside the unit circle), there exists a critical value of detection probability $p_{d}$, below which the modified Riccati equation diverges.

Simulation results were taken concerning the modified Riccati equation. Both the classical and the proposed algorithms were implemented in order to solve the modified Riccati equation. Various stable as well as unstable models were considered. The following results were confirmed.

(i) Both algorithms have the same behavior. If the system is stable (all eigenvalues of $F$ lie inside the unit circle), then the modified Riccati equation always converges: there always exists a steady-state solution. If the system is unstable (there is at least one eigenvalue of $F$ that lies strictly outside the unit circle), then there exists a critical value of detection probability $p_{d}$, below which the modified Riccati equation diverges.

(ii) The critical value of detection probability $p_{d}$ increases in a logarithmic way as the maximum absolute eigenvalue of $F$ increases, that is, the system becomes more unstable. Figure 2 depicts the relation between the system stability and the modified Riccati equation convergence.

(iii) In the special case where the maximum absolute eigenvalue of $F$ lies in the unit circle, the critical value of detection probability takes its minimum value of the order of 0.04 .

(iv) The maximum absolute eigenvalue of $F$, below which the modified Riccati equation always diverges, is of the order of 10 .

\section{References}

[1] T. E. Fortmann, Y. Bar-Shalom, M. Scheffe, and S. Gelfand, "Detection thresholds for tracking in clutter-a connection between estimation and signal processing," IEEE Transactions on Automatic Control, vol. 30, no. 3, pp. 221-229, 1985.

[2] Y. Boers and H. Driessen, "Tracking performance prediction and evaluation: the modified Riccati equation," in Signal and Data Processing of Small Targets (SPIE '05), August 2005.

[3] Y. Boers and H. Driessen, "Results on the modified Riccati equation: target tracking applications," IEEE Transactions on Aerospace and Electronic Systems, vol. 42, no. 1, pp. 379-384, 2006.

[4] A. S. Chhetri, D. Morrell, and A. Papandreou-Suppappola, "Nonmyopic sensor scheduling and its efficient implementation for target tracking applications," Eurasip Journal on Applied Signal Processing, vol. 2006, Article ID 31520, 2006.

[5] A. Farina, A. Di Lallo, L. Timmoneri, T. Volpi, and B. Ristic, "CRLB and ML for parametric estimate: new results," Signal Processing, vol. 86, no. 4, pp. 804-813, 2006.

[6] M. Hernandez, B. Ristic, A. Farina, and L. Timmoneri, "A comparison of two Cramér-Rao bounds for nonlinear filtering with $P_{d}<1$," IEEE Transactions on Signal Processing, vol. 52, no. 9, pp. 2361-2370, 2004. 
[7] C. O. Savage and B. F. La Scala, "Sensor management for tracking smart targets," Digital Signal Processing, vol. 19, no. 6, pp. 968-977, 2009.

[8] B. Ristic, S. Arulampalam, and N. Gordon, Beyond the Kalman Filter-Particle Filters for Tracking Applications, Artech House, Boston, Mass, USA, 2002.

[9] B. Sinopoli, L. Schenato, M. Franceschetti, K. Poolla, M. I. Jordan, and S. S. Sastry, "Kalman filtering with intermittent observations," IEEE Transactions on Automatic Control, vol. 49, no. 9, pp. 1453-1464, 2004.

[10] J. H. Zwaga and H. Driessen, "Tracking performance constrained MFR parameter control: applying constraints on prediction accuracy," in Proceedings of the 8th International Conference on Information Fusion (FUSION '05), vol. 1, p. 6, July 2005.

[11] B. D. O. Anderson and J. B. Moore, Optimal Filtering, Dover Publications, New York, NY, USA, 2005.

[12] R. E. Kalman, "A new approach to linear filtering and prediction problems," Journal of Basic Engineering, Transactions on the ASME, vol. 82, pp. 34-45, 1960.

[13] H. Nguyen, J. Zhang, and B. Raghothaman, "A Kalmanfilter approach to equalization of CDMA downlink channels," Eurasip Journal on Applied Signal Processing, vol. 2005, no. 5, Article ID 750826, 2005.

[14] N. Assimakis, A. Kechriniotis, S. Voliotis, F. Tassis, and M. Kousteri, "Analysis of the time invariant Kalman filter implementation via general Chandrasekhar algorithm," International Journal of Signal and Imaging Systems Engineering, vol. 1, no. 1, pp. 51-57, 2008. 

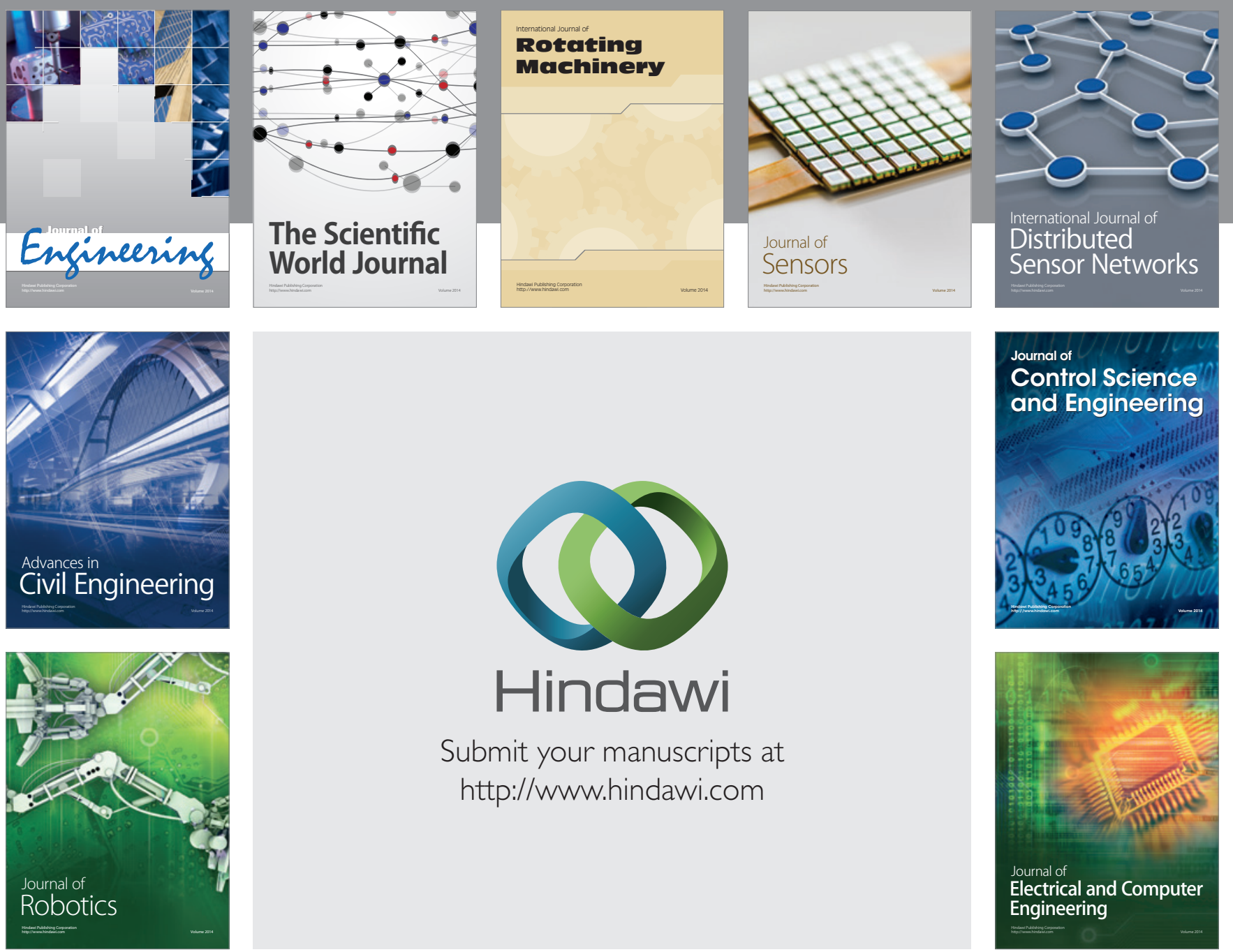

Submit your manuscripts at

http://www.hindawi.com
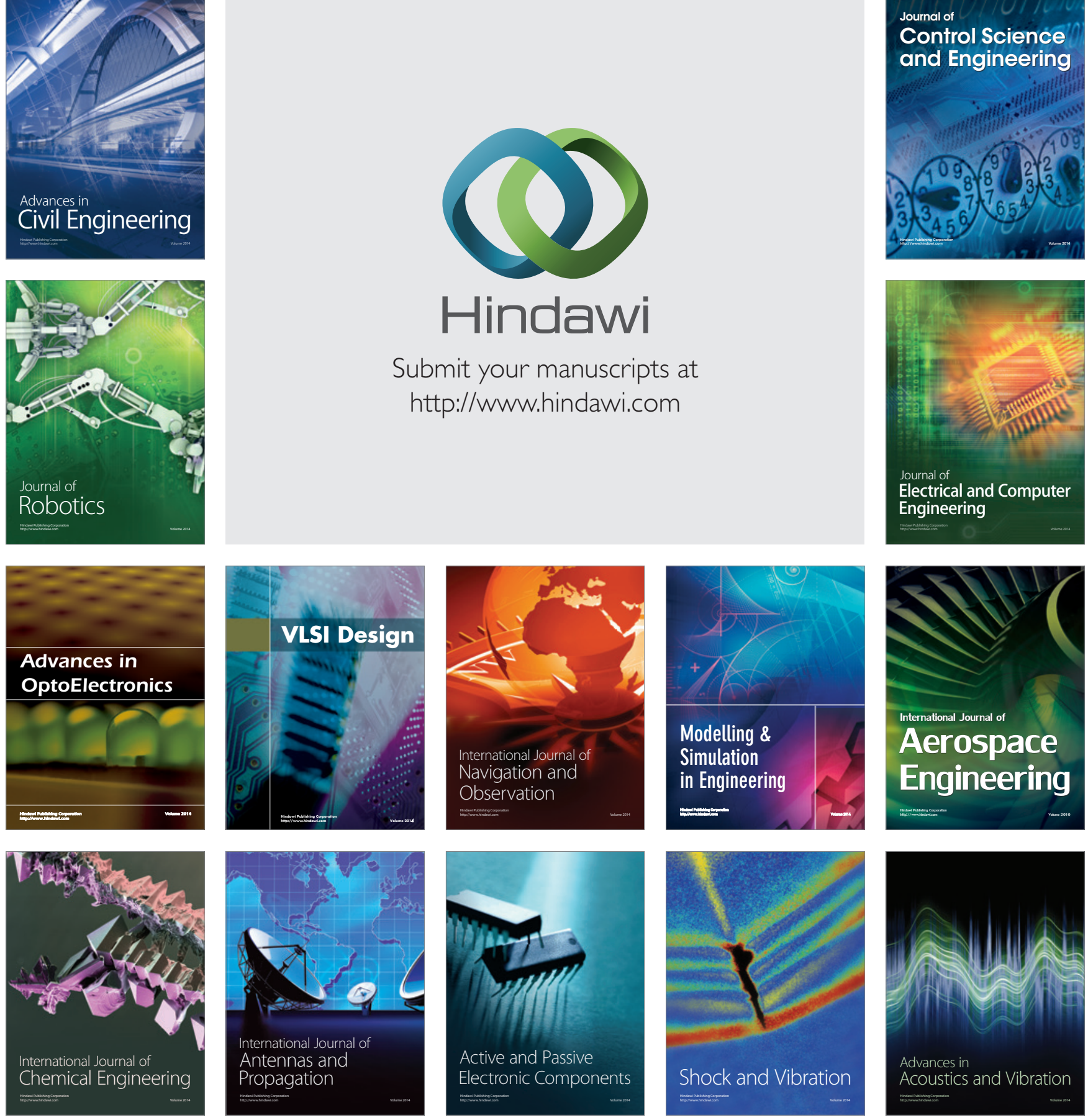